\title{
CORRESPONDENCE
}

\section{Ocular Changes During Pregnancy}

by PD Dr. med. Friederike Mackensen, Dr. med. Wolfgang E. Paulus, Dr. med. Regina Max, PD Dr. med. Thomas Ness in issue 33-34/2014

\section{Further Specification Required}

The authors deserve high appreciation for their comprehensive approach to the subject (1). The clinical relevance demands, however, that the following items are specified to some extent:

- The authors state that during pregnancy diabetic retinopathy can deteriorate by up to $55 \%$. This (maximum) value should be put in perspective in view of the specific treatment conditions in Germany.

- Among the risk factors for deterioration of diabetic retinopathy the authors cite the regular blood glucose monitoring required before pregnancy. 94.8\% of all women with type 1 diabetes receive intensified conventional treatment (ICT) which requires regular blood glucose monitoring. This means that almost every woman with type 1 diabetes who is receiving intensified conventional treatment runs the risk of deteriorating. The parameter "regular blood glucose monitoring before pregnancy" is therefore no sufficiently predictive factor.

- The proposal to prefer bevacizumab (complete antibody) to ranibizumab (Fab fragment) is not justified, as the literature reports miscarriages during bevacizumab treatment. In the cited article, it is early pregnancy, but not the anti-VEGF antibody that causes miscarriages. Therefore, any anti-VEGF therapy should be prohibited during early pregnancy. Thus far, no VEGF inhibitor has been approved for the treatment of proliferative diabetic retinopathy.

- The recommendation to examine the retinal fundus one year after delivery in women with type 1 diabetes and diabetic retinopathy who are closely monitored is not supported by the guidelines. Since the justification for close monitoring is pre-existing retinopathy, the post partum screening intervals should be conducted according to the level of pre-existing retinopathy (mild-moderate non-proliferative diabetic retinopathy 6 months; severe non-proliferative diabetic retinopathy: 3 months).

DOI: 10.3238/arztebl.2015.0194a

\section{REFERENCES}

1. Mackensen F, Paulus WE, Max R, Ness T: Ocular changes during pregnancy. Dtsch Arztebl Int 2014; 111: 567-76.

Prof. Dr. med. Hans-Peter Hammes Universitätsmedizin Mannheim hpmh@gmx.de

Conflict of interest statement

The author declares that no conflict of interest exists.
In Reply:

We thank Professor Hammes for his comments relating to our CME article in Deutsches Ärzteblatt. We wish to address the individual items one by one, as follows:

- Unfortunately there are no data for Germany. The data we presented come from large, partly prospective studies from the US.

- The authors refer to control/monitoring of diabetic retinopathy, not that of blood glucose, even if those two factors are not mutually independent. The decisive factor is not the blood glucose concentration before pregnancy but the extent of diabetic retinopathy. This was what we described in the article. The studies further mention the need for blood glucose monitoring as a separate risk factor. Professor Hammes rightly points out that if this was taken at face value, all patients receiving intensified conventional treatment (ICT) had an increased risk. However, this should always been seen in the total context and relate primarily to the extent of retinopathy before or at the start of pregnancy.

- Professor Hammes is completely right in this point: the VEGF inhibitors are not licensed for the treatment of proliferative diabetic retinopathy. We commented on the treatment of diabetic macular edema. This is usually not required during pregnancy. Our comments relate to the use of VEGF inhibitors in other indications with acute risk to visual acuity (for example myopic neovascularization of the macula). The use of these substances will need to be decided on an individual basis in this setting.

- The recommended control examination after a year (according to the guidelines of the American Diabetes Association) is suggested in settings where no other, closer control intervals are adhered to because of the retinopathy. The article explains this in a way that may give rise to misunderstandings. We thank Professor Hammes for pointing this out

DOI: 10.3238/arztebl.2015.0194b

\section{REFERENCES}

1. Mackensen F, Paulus WE, Max R, Ness T: Ocular changes during pregnancy. Dtsch Arztebl Int 2014; 111: 567-76.

PD Dr. med. Friederike Mackensen

Universitäts-Augenklinik Heidelberg

mackensen@uveitiszentrum.de

PD Dr. med. Thomas Ness

Klinik für Augenheilkunde, Universitätsklinikum Freiburg

Conflict of interest statement

PD Dr. Mackensen has served as a paid consultant for Merck Serono and has received payment for continuing medical education events from Heidelberg Engineering.

PD Dr. Ness has served as a paid consultant for Abbvie. He has received reimbursement of meeting participation fees and travel and accommodation expenses from Bayer Health Care, as well as payment for continuing medica education events form Abbvie and Novartis and for the performance of clinica trials on behalf of Novartis, Santen, Abbvie, Sanofi, Allergan, and pSivida corp. 